\title{
Efeito do Tipo de Chapa de Partículas nas Propriedades Físicas e Mecânicas
}

\author{
Carla Priscilla Távora Cabral $^{1}$, Bárbara Luísa Corradi Pereira ${ }^{1}$, \\ Danila Morais de Carvalho ${ }^{1}$, Aylson Costa Oliveira ${ }^{1}$, Benedito Rocha Vital ${ }^{1}$, \\ Cláudia Márcia Gomes ${ }^{1}$, Angélica Cássia Oliveira Carneiro ${ }^{1}$ \\ ${ }^{1}$ Departamento de Engenharia Florestal, Universidade Federal de Viçosa - UFV, Viçosa/MG, Brasil
}

\begin{abstract}
RESUMO
Este trabalho teve como objetivo avaliar chapas de partículas do tipo aglomerado convencional, chapas tipo Medium Density Particleboard (MDP) e as do tipo aglomerado com inclusão laminar. As chapas avaliadas foram fabricadas com partículas de Acacia mangium e no aglomerado com inclusão laminar utilizaram-se lâminas de Schizolobium amazonicum. O aglomerado convencional e o aglomerado com inclusão laminar apresentaram umidade de equilíbrio higroscópico estatisticamente superior ao MDP. O aglomerado com inclusão laminar apresentou maior índice de inchamento em espessura após duas horas de imersão em água, valores de módulo de ruptura em flexão estática maiores e maior resistência ao arrancamento de parafuso. As chapas do tipo MDP apresentaram maior resistência à tração perpendicular. Não foram observadas diferenças significativas nas demais propriedades. Conclui-se que a inclusão laminar melhorou o módulo de ruptura e o arrancamento de parafuso, enquanto a utilização de partículas finas nas faces melhorou as propriedades físicas das chapas.
\end{abstract}

Palavras-chave: aglomerado convencional, painel de partículas de média densidade, inclusão laminar.

\section{Effect of Particleboard Type in Physical and Mechanical Properties}

\begin{abstract}
This study aimed to evaluate physical and mechanical properties of conventional agglomerate, medium density particleboard and agglomerate with laminate inclusion. Particleboards were made of Acacia mangium particles and laminas of Schizolobium amazonicum were used to produce agglomerate with laminate inclusion. Conventional agglomerate and agglomerate with laminate inclusion showed hygroscopic equilibrium moisture statistically superior to MDP. Agglomerate with laminate inclusion showed the highest rate of thickness swelling after 2 hours of immersion in water, the highest modulus of rupture in bending strength and the best screw withdraw resistance. MDP presented the highest mean value of board internal bond strength. There were no significant differences in other evaluated properties. It was concluded that laminate inclusion improved modulus of rupture and screw withdraw resistance while the use of fine particles on the faces improved physical properties of the particleboards.
\end{abstract}

Keywords: conventional agglomerate, medium density particleboard, laminate inclusion. 


\section{INTRODUÇÃO}

Chapas de partículas de madeira aglomerada são produtos fabricados com partículas de material lignocelulósico, geralmente madeira, aglutinados com adesivos sintéticos, sendo o conjunto prensado a quente, o que promove o adensamento do colchão e acelera as reações de polimerização do adesivo (Iwakiri, 2005).

No Brasil, o aglomerado sempre foi um painel com algumas limitações tecnológicas, que começou a ser melhorado e sua demanda interna cresceu, mas seu estigma de inferioridade ainda predominava (Mendoza, 2010). Ainda de acordo com essa autora, nos anos 2005 e 2006, as empresas brasileiras começaram a substituí-lo, gradativamente, por um produto que recebeu o nome de MDP (Medium Density Particleboard), ou painel de partículas de média densidade. Segundo a Indústria Brasileira de Árvores (IBÁ, 2015), a produção anual de MDP em 2014 foi de 3,2 milhões de $\mathrm{m}^{3}$, sendo que 95\% dos painéis produzidos no país têm como destino o mercado nacional.

Segundo Bruno (2007), o MDP pertence a uma geração de painéis de partículas de média densidade, apresentando melhores propriedades do que os painéis fabricados até então, sendo produzido com três camadas. A camada interna formada com partículas mais grossas e as camadas externas, com partículas mais finas. O mesmo autor menciona ainda que, devido ao seu processo de produção, as camadas superficiais apresentam densidade mais elevada, o que assegura ao painel propriedades mecânicas otimizadas e um excelente acabamento.

Dentre os trabalhos de pesquisa desenvolvidos na área de compósitos de madeira merece destaque a inclusão de lâminas na estrutura do painel, denominado aglomerado com inclusão laminar. Nesse processo, lâminas de madeira são aderidas na face e contraface, melhorando a resistência da chapa e minimizando as limitações de uso ocasionadas pela instabilidade dimensional dos produtos reconstituídos (Iwakiri et al., 1999; Mendoza, 2010). Na sua confecção, pode-se utilizar qualquer parte da árvore ou da madeira que seria desprezada, isso é um atrativo grande na área de industrialização, solucionando, em parte, o problema ambiental causado pelos resíduos, além de agregar valor ao produto final.

Os painéis de madeira reconstituída apresentam grande versatilidade, suprindo o uso da madeira sólida para os mais diversos fins. Além de apresentarem vantagem na redução do custo da matéria-prima, eles também viabilizam a utilização dos mais variados tipos de resíduos, dependo da finalidade do painel.

Com base no exposto, o presente trabalho objetivou avaliar as propriedades físicas e mecânicas de chapas do tipo aglomerado convencional, chapas MDP e de aglomerado com inclusão laminar produzidos com partículas de Acacia mangium Willd e lâminas de paricá (Schizolobium amazonicum Hurber).

\section{MATERIAL E MÉTODOS}

O experimento foi realizado no Laboratório de Painéis e Energia da Madeira (LAPEM) da Universidade Federal de Viçosa, estado de Minas Gerais.

Foram produzidas chapas de partículas do tipo aglomerado convencional, Medium Density Particleboards (MDP) e de aglomerado com inclusão laminar, conforme a Figura 1.

As partículas utilizadas na produção das chapas foram produzidas a partir de cavacos de Acacia mangium, que foram processados em um moinho de martelos equipado com peneira de $2 \times 22,5 \mathrm{~mm}$. Posteriormente realizou-se a classificação em uma peneira manual com malha de $1,0 \times 1,0 \mathrm{~mm}$. As lâminas de paricá foram obtidas de empresas laminadoras localizadas no estado do Pará e apresentavam, em média, $3 \mathrm{~mm}$ de espessura, largura de $40 \mathrm{~cm}$ e comprimento de $40 \mathrm{~cm}$.

As partículas e as lâminas foram secas em estufa de circulação forçada à $65^{\circ} \mathrm{C}$ até atingirem $3 \%$ de umidade. $\mathrm{O}$ valor médio da densidade das partículas de Acacia mangium foi igual a $0,386 \mathrm{gcm}^{-3} \mathrm{e}$ o das lâminas de paricá, igual a $0,324 \mathrm{gcm}^{-3}$.

Para a produção das chapas utilizou-se o adesivo ureia-formaldeído, cujas principais propriedades obtidas em laboratório estão na Tabela 1 .

$\mathrm{O}$ adesivo foi aplicado sobre as partículas com o auxílio de uma encoladeira do tipo tambor rotatório, a qual continha um bico aspersor integrado ao compressor. A quantidade de adesivo utilizada foi de $8 \%$ sobre a massa seca de partículas. O colchão de partículas foi formado numa caixa vazada separada por placas de alumínio e pré-prensado manualmente para melhor acomodação das partículas. A inclusão das lâminas foi feita na face e contraface do aglomerado, sem aplicação de adesivo nas lâminas, e sua prensagem ocorreu simultaneamente com a do colchão de partículas. 


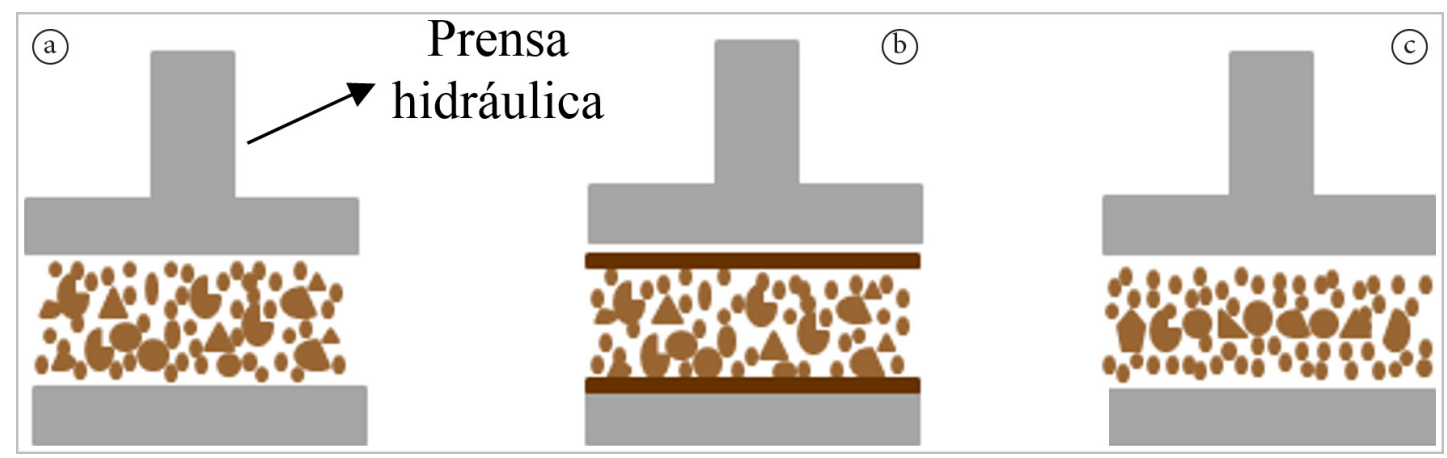

Figura 1. Tipos de chapas produzidas: (a) aglomerado convencional; (b) aglomerado com inclusão laminar; e (c) Medium Density Particleboard (MDP)

Figure 1. Types of particleboard: (a) conventional particleboard; (b) com-ply; and (c) medium density particleboard (MDP).

Tabela 1. Propriedades do adesivo ureia-formaldeído utilizado na produção das chapas.

Table 1. Properties of urea-formaldehyde adhesive used in the particleboards production.

\begin{tabular}{cccccc}
\hline Adesivo & $\begin{array}{c}\text { Massa específica } \\
\left(\mathbf{g ~ c m}^{-3}\right)\end{array}$ & $\begin{array}{c}\text { Teor de sólidos } \\
(\%)\end{array}$ & $\mathbf{p H}$ & $\begin{array}{c}\text { Viscosidade } \\
(\mathbf{c p})\end{array}$ & $\begin{array}{c}\text { Tempo de } \\
\text { gelatinização } \\
(\mathbf{s e g})\end{array}$ \\
\hline ureia-formaldeído & 1,31 & 62,67 & 7,96 & 1077 & 167 \\
\hline
\end{tabular}

A prensagem foi realizada numa prensa hidráulica, com pressão de $32 \mathrm{kgfcm}^{-2}$, temperatura dos pratos metálicos de $170{ }^{\circ} \mathrm{C}$ e tempo de prensagem de 8 minutos, contados a partir do fechamento da prensa. As chapas foram fabricadas com dimensões nominais de $40 \times 40 \times 1 \mathrm{~cm}$. A taxa de compactação foi igual a 1,8

As chapas de MDP foram feitas usando-se procedimento semelhante ao do aglomerado convencional, com exceção da distribuição das partículas na caixa formadora. Para formar o colchão do MDP foram feitas três pesagens para a quantidade de partículas a serem distribuídas na caixa formadora, as que iriam compor o miolo (partículas de maior dimensão) e as partículas que iriam compor as faces da chapa (partículas de menor dimensão).

Depois de prensadas, as chapas foram acondicionadas à temperatura ambiente até atingirem umidade de equilíbrio. Posteriormente foram retirados os corpos de prova, para os ensaios físicos e mecânicos. Os testes foram realizados após a climatização dos corpos de prova a $65 \pm 5 \%$ de umidade relativa (UR) e temperatura de $20 \pm 3{ }^{\circ} \mathrm{C}$, segundo as Normas da Associação Brasileira de Normas Técnicas - ABNT NBR 14.810-2 (ABNT, 2013).

De cada painel foram retiradas amostras para determinação das propriedades físicas (umidade; absorção de água e inchamento em espessura após duas horas de imersão em água; massa específica) e mecânicas (tração perpendicular; flexão estática; arrancamento de parafuso perpendicular à superfície da chapa; dureza Janka; compressão longitudinal).

O experimento foi instalado seguindo-se um delineamento inteiramente casualizado, com três tratamentos (aglomerado convencional, MDP e aglomerado com inclusão laminar) e três repetições (chapas) por tratamento, totalizando nove chapas. Os testes físicos e mecânicos foram realizados em triplicata. Os resultados foram submetidos à análise de variância (ANOVA). Quando se estabeleceram diferenças significativas, os tratamentos foram comparados entre si, por meio do teste de Tukey a 5\% de significância.

\section{RESULTADOS E DISCUSSÃO}

\subsection{Propriedades físicas}

As médias para umidade, absorção de água após duas horas e inchamento em espessura das diferentes chapas estão apresentadas na Tabela 2.

Após equilíbrio a uma temperatura de $20 \pm 3{ }^{\circ} \mathrm{Ce}$ a $65 \pm 5 \%$ de umidade relativa, as chapas apresentaram umidade média variando de 8,13 a 9,58\%. Verificou-se 
que o aglomerado convencional e o aglomerado com inclusão laminar apresentaram um teor de umidade estatisticamente superior ao MDP. Os valores encontrados estão em concordância com o trabalho de Bianche et al. (2012), que encontraram valores médios de $8,8 \%$ de umidade para painéis de partículas de Schizolobium amazonicum. Segundo a ABNT NBR 14.810-2 (ABNT, 2013), para comercialização, o teor médio de umidade de chapas de madeira aglomerada deve estar entre 5 e $11 \%$. Portanto, as chapas produzidas atenderam ao requisito dessa norma.

Observou-se que a absorção de água após duas horas de imersão das chapas dos tipos aglomerado convencional e MDP absorveram menor quantidade de água em relação ao aglomerado com inclusão laminar. A maior absorção de água por esse tipo de painel pode ser explicada pelas lâminas na face e contraface.

A absorção de água foi superior ao permitido pela norma DIN 68.761 (1)-1961 (3) (DIN, 1971), conforma a qual os valores devem estar abaixo de $10 \%$. Já a norma pertinente da ABNT não estabelece valores máximos ou mínimos de absorção de água por imersão.

Ainda de acordo com a Tabela 2, o inchamento em espessura foi maior no aglomerado com inclusão laminar e menor no aglomerado convencional, assim como no teste de absorção de água após duas horas. O MDP apresentou um inchamento intermediário, não diferindo estatisticamente dos outros dois tipos de chapa.

De acordo com a norma ABNT NBR 14.810-2 (ABNT, 2013), o inchamento em espessura deve ser inferior a $8 \%$ para duas horas de imersão em água, portanto, dos painéis avaliados, apenas o aglomerado com inclusão laminar não atendeu aos requisitos da norma para tal propriedade.

O tipo de chapa não teve efeito significativo sobre a massa específica $(\mathrm{p}<0,05)$, obtendo-se uma média geral de $680 \mathrm{kgm}^{-3}$. Esse valor está de acordo com o encontrado por Bianche et al. (2012), que aferiram densidade de painéis de partículas de Schizolobium amazonicum igual a $700 \mathrm{kgm}^{-3}$. De acordo com a norma ABNT NBR 14.810-2 (ABNT, 2013), são classificados como painéis de média massa específica.

\subsection{Propriedades mecânicas}

Na Tabela 3 estão os valores médios das propriedades mecânicas que apresentaram diferenças significativas $(\mathrm{p}<0,05)$.

As chapas de MDP apresentaram os maiores valores de resistência à tração perpendicular, seguidas

Tabela 2. Valores médios de umidade, absorção de água e inchamento de espessura em função do tipo de chapa. Table 2. Mean values of moisture content, water absorption and thickness swelling, depending on the particleboard type.

\begin{tabular}{lccc}
\multicolumn{1}{c}{ Chapa } & \multicolumn{3}{c}{ Propriedades físicas } \\
\cline { 2 - 4 } & Umidade (\%) & $\begin{array}{c}\text { Absorção de } \\
\text { água }-2 \text { horas (\%) }\end{array}$ & $\begin{array}{c}\text { Inchamento em } \\
\text { espessura (\%) }\end{array}$ \\
\hline Aglomerado convencional & $9,58 \mathrm{a}$ & $14,31 \mathrm{~b}$ & $6,43 \mathrm{~b}$ \\
Aglomerado com inclusão laminar & $9,40 \mathrm{a}$ & $27,84 \mathrm{a}$ & $11,10 \mathrm{a}$ \\
MDP & $8,13 \mathrm{~b}$ & $11,04 \mathrm{~b}$ & $7,28 \mathrm{ab}$ \\
\hline
\end{tabular}

Médias seguidas por letras iguais nas colunas não diferem estatisticamente pelo teste de Tukey $(\mathrm{p}<0,05)$.

Tabela 3. Valores médios de tração perpendicular, módulo de ruptura (MOR) em flexão estática e arrancamento de parafuso perpendicularmente à superfície da chapa em função do tipo de chapa.

Table 3. Mean values of board internal bond strength, modulus of rupture and screw withdraw resistance, depending on the particleboard type.

\begin{tabular}{lccc} 
& \multicolumn{3}{c}{ Propriedades mecânicas } \\
\cline { 2 - 4 } \multicolumn{1}{c}{ Chapa } & $\begin{array}{c}\text { Tração perpendicular } \\
(\mathbf{M P a})\end{array}$ & $\begin{array}{c}\text { Flexão estática MOR } \\
(\mathbf{M P a})\end{array}$ & $\begin{array}{c}\text { Arrancamento de } \\
\text { parafuso (N) }\end{array}$ \\
\hline Aglomerado convencional & $0,86 \mathrm{ab}$ & $11,59 \mathrm{~b}$ & $787,80 \mathrm{ab}$ \\
\hline Aglomerado com inclusão laminar & $0,19 \mathrm{~b}$ & $22,72 \mathrm{a}$ & $1068,92 \mathrm{a}$ \\
MDP & $1,21 \mathrm{a}$ & $14,89 \mathrm{ab}$ & $629,26 \mathrm{~b}$ \\
\hline
\end{tabular}

Médias seguidas por letras iguais nas colunas não diferem estatisticamente pelo teste de Tukey $(\mathrm{p}<0,05)$. 
pelas chapas de aglomerado convencional. As chapas de aglomerado com inclusão laminar apresentaram resistência à tração perpendicular inferior ao mínimo estipulado pelas normas, ocorrendo delaminação entre a lâmina e o miolo do painel. Isso se deve provavelmente à insuficiência de adesivo na superfície das lâminas, uma vez que nelas não foi aplicado adesivo.

Mendoza (2010), ao avaliar painéis do tipo aglomerado com e sem inclusão laminar, verificou que a tração perpendicular em painéis com inclusão laminar é superior, porém, a autora aplicou adesivo nas lâminas da face e contraface.

À exceção do aglomerado, os demais painéis apresentaram valores médios de tração perpendicular superiores ao mínimo exigido pela norma ABNT NBR 14.810-2 (ABNT, 2013), 0,40 MPa, para painéis com espessura entre 8 e $14 \mathrm{~mm}$, na qual se enquadram os painéis produzidos para o presente estudo.

O módulo de ruptura em flexão estática foi inferior para o aglomerado convencional, sendo que a utilização de partículas menores nas superfícies (MDP) ou inclusão de lâminas nas faces melhoraram tal propriedade. O aglomerado com inclusão laminar obteve os maiores resultados para módulo de ruptura, atendendo o valor mínimo estipulado pela norma ABNT NBR 14.810-2 (ABNT, 2013), que é de $16 \mathrm{MPa}$.

O MDP apresentou o menor valor médio para arrancamento de parafuso perpendicularmente à superfície da chapa e o aglomerado com inclusão laminar apresentou o maior valor médio para a mesma propriedade. O aglomerado convencional apresentou valor médio intermediário, não diferindo estatisticamente dos demais.

Dentre as propriedades mecânicas avaliadas, não houve efeito significativo entre os tipos de painel $(\mathrm{p}<0,05)$ para dureza Janka, resistência à compressão longitudinal e módulo de elasticidade em flexão.

A dureza Janka, que mede a resistência superficial, foi, em média, igual a 33,05 MPa. O valor mínimo de dureza Janka requerido pela norma ABNT NBR 14.810-2 (ABNT, 2013) é de 1,0 MPa. Assim, os painéis avaliados no presente estudo alcançaram valores superiores aos estipulados por tal norma.
O valor médio de compressão longitudinal foi igual a 10,35 MPa. Para tal propriedade não há especificação normatizada do valor mínimo aceitável para comercialização. Cabral et al. (2007), ao avaliar as propriedades de chapas de madeira aglomerada confeccionadas com partículas geradas de maravalha e flocos de Eucalyptus grandis e Eucalyptus cloeziana, encontrou valores médios de compressão longitudinal de 56,72 a 88,39 MPa, respectivamente.

O valor médio de módulo de elasticidade em flexão estática para os três tipos de chapas, aglomerado convencional, aglomerado com inclusão laminar e MDP foi de $1.658,75 \mathrm{MPa}$. O valor médio é inferior ao encontrado por Bianche et al. (2012), 2.955,69 MPa, para painéis de partículas de Schizolobium amazonicum.

\section{CONCLUSÕES}

- A inclusão laminar na composição estrutural dos painéis aglomerados melhorou o módulo de ruptura (MOR) e arrancamento de parafuso perpendicularmente à superfície da chapa.

- A utilização de partículas mais finas nas faces, como foi feito no MDP, melhorou as propriedades físicas (umidade, absorção de água e inchamento em espessura - duas horas de imersão) em relação ao aglomerado tradicional, além de apresentar também resultados superiores para tração perpendicular.

\section{AGRADECIMENTOS}

Os autores agradecem à Universidade Federal de Viçosa, ao Departamento de Engenharia Florestal e ao Laboratório de Painéis e Energia da Madeira.

\section{STATUS DA SUBMISSÃO}

Recebido: 19 fev., 2013

Aceito: 2 nov., 2015

\section{AUTOR(ES) PARA CORRESPONDÊNCIA}

\section{Bárbara Luísa Corradi Pereira}

Departamento de Engenharia Florestal, Universidade Federal de Viçosa - UFV, CEP 36570-000, Viçosa, MG, Brasil e-mail: babicorradi@gmail.com 


\section{REFERÊNCIAS}

Associação Brasileira de Normas Técnicas - ABNT. NBR 14810-2: painéis de partículas de média densidade: parte 2: requisitos e métodos de ensaio. Rio de Janeiro; 2013.

Bianche JJ, Carneiro ACO, Vital BR, Pereira FA, Santos RC, Soratto DN. Propriedades de painéis aglomerados fabricados com partículas de eucalipto (Eucalyptus urophylla), paricá (Schizolobium amazonicum) e vassoura (Sida spp.). Cerne 2012; 18(4): 623-630. http://dx.doi. org/10.1590/S0104-77602012000400012.

Bruno EG. Painéis facilitam usinagem na fabricação moveleira. Revista da Madeira 2007; 18(109): 48-50.

Cabral CP, Vital BR, Della Lucia RM, Pimenta AS. Propriedades de chapas de aglomerado confeccionadas com misturas de partículas de Eucalyptus spp. e Pinus elliottii. Revista Árvore 2007; 31(5): 897-905. http://dx.doi. org/10.1590/S0100-67622007000500014.

Deutsches Institut für Normung - DIN. DIN 68761 (1)1961 (3): specifications for particleboards. Holtz; 1971.

Indústria Brasileira de Árvores - IBÁ. Relatório da Indústria Brasileira de Árvores. São Paulo; 2015.

Iwakiri S, Costa AF, Klitzke RJ, Nielsen IR, Alberti RAR, Niefe I. Produção de chapas de madeira aglomerada de Pinus elliottii Engelm com inclusão laminar. Cerne 1999; 5(2): 86-94.

Iwakiri S. Painéis de madeira reconstituída. 1. ed. Curitiba: FUPEF, 2005.

Mendoza ZMSH. Efeito da inclusão laminar nas propriedades de painéis aglomerados fabricados com resíduos da indústria laminadora [tese]. Viçosa: Departamento de Engenharia Florestal, Universidade Federal de Viçosa: 2010. 